\title{
Body height and waist circumference of young Swiss men as assessed by 3D laser-based photonic scans and by manual anthropometric measurements
}

\author{
Claudia Beckmann ${ }^{1}$, Lafi Aldakak ${ }^{1}$, Patrick Eppenberger ${ }^{1}$, Frank Rühli $^{1}$, Kaspar Staub $^{1}$, Nicole Bender ${ }^{\text {Corresp. } 1}$ \\ ${ }^{1}$ Institute of Evolutionary Medicine, University of Zurich, Zurich, Switzerland \\ Corresponding Author: Nicole Bender \\ Email address: nicole.bender@iem.uzh.ch
}

Overweight and obesity are considered among the major health concerns worldwide. The body mass index is a frequently used measure for overweight and obesity and is associated with common non-communicable diseases such as diabetes type II, cardiovascular diseases and certain cancers. However, the body mass index does not account for the distribution of body fat and relative fat to muscle mass. 3D laser-based photonic full body scans provide detailed information on various body circumferences, surfaces, and volumes as well as body height and weight (using an integrated scale). In the literature, body scans showed good feasibility, reliability, and validity, while also demonstrating a good correlation with health parameters linked to the metabolic syndrome. However, systematic differences between body scan derived measurements and manual measurements remain an issue. This study aimed to assess these systematic differences for body height, waist circumference, and body mass index using crosssectional data from a homogenous sample of 52 young Swiss male volunteers. In addition to 3D laser-based photonic full body scans and correlative manual measurements, body fat distribution was assessed through bioelectrical impedance analysis. Overall, an excellent correlation was found between measurements of waist circumference and body mass index, and good correlation between body mass index and total fat mass, as well as between waist circumference and visceral fat mass as assessed by bioelectrical impedance analysis. Volunteers were shorter in height measured by body scan when compared to manual measurements. This systematic difference became smaller when volunteers stood in the scanner in a completely upright position with their feet together. Waist circumference was slightly smaller for manual measurements than for body scan derived values. This systematic difference was larger in overweight volunteers compared to leaner volunteers. 
1 Body height and waist circumference of young Swiss men as assessed by 3D laser-based

2 photonic scans and by manual anthropometric measurements

3

4

5 Claudia Beckmann ${ }^{1}$; Lafi Aldakak ${ }^{1}$; Patrick Eppenberger ${ }^{1}$; Frank Rühli ${ }^{1}$; Kaspar Staub ${ }^{1 *}$; Nicole 6 Bender $^{1 * \S}$

7

$8{ }^{1}$ Institute of Evolutionary Medicine, University of Zurich, Winterthurerstrasse 190, 8057 Zurich, 9 Switzerland

10

$11 *$ These authors contributed equally to this work: Last authorship

$12 \S$ Corresponding author: Nicole Bender, nicole.bender@iem.uzh.ch

13

14

15

Short title: Height and WC: scanner vs manual measure

16

17

18

19

20 


\section{Abstract}

22

23 Overweight and obesity are considered among the major health concerns worldwide. The body

24 mass index is a frequently used measure for overweight and obesity and is associated with

25 common non-communicable diseases such as diabetes type II, cardiovascular diseases and 26 certain cancers. However, the body mass index does not account for the distribution of body fat

27 and relative fat to muscle mass. 3D laser-based photonic full body scans provide detailed

28 information on various body circumferences, surfaces, and volumes as well as body height and

29 weight (using an integrated scale). In the literature, body scans showed good feasibility,

30 reliability, and validity, while also demonstrating a good correlation with health parameters

31 linked to the metabolic syndrome. However, systematic differences between body scan derived

32 measurements and manual measurements remain an issue. This study aimed to assess these

33 systematic differences for body height, waist circumference, and body mass index using cross-

34 sectional data from a homogenous sample of 52 young Swiss male volunteers. In addition to 3D

35 laser-based photonic full body scans and correlative manual measurements, body fat distribution

36 was assessed through bioelectrical impedance analysis. Overall, an excellent correlation was

37 found between measurements of waist circumference and body mass index, and good correlation

38 between body mass index and total fat mass, as well as between waist circumference and visceral

39 fat mass as assessed by bioelectrical impedance analysis. Volunteers were shorter in height

40 measured by body scan when compared to manual measurements. This systematic difference

41 became smaller when volunteers stood in the scanner in a completely upright position with their

42 feet together. Waist circumference was slightly smaller for manual measurements than for body

43 scan derived values. This systematic difference was larger in overweight volunteers compared to

44 leaner volunteers. 


\section{Introduction}

47 Overweight and obesity are major global health concerns, and worldwide more than 1.9 billion

48

49

50

51

52

53

54

55

56

57

58

59

60

61

62

63

64

65

66

67

68

69

70

71

72

73

74

75

adults were overweight in 2016 (WHO 2017). The body mass index (BMI, weight in kilograms

divided by squared height in meters) is a frequently used measure for overweight and obesity and is associated with common non-communicable diseases such as diabetes type II, cardiovascular diseases and certain cancers (WHO 2018). However, the BMI does not account for the distribution of body fat and relative fat to muscle mass. Waist circumference (WC) and waist-tohip ratio are considered to be better than the BMI to predict CVD (Kjaer et al. 2015; WHO 2008). However, it is still unclear which measures best correlate with disease risk (Ashwell et al. 2012; Kahn and Bullard 2016; Lam et al. 2015). Other techniques used at a population level like Bioimpedance Analysis (BIA) offer and quick and safe measurement of height, weight, BMI, total fat mass, visceral fat mass, and muscle mass, but measures like WC have to be taken classically with a tape and values have to be entered manually in the BIA machine. Furthermore, BIA seems to be less precise in measuring visceral fat mass than other techniques such as the reference standards, computed tomography (CT) and magnetic resonance imaging (MRI) (Murphy et al. 2019). Other circumferences and ratios derived from them are not assessed. Such measurements have therefore their own limitations and new approaches to quantify obesity and categorize the corresponding health risks are therefore necessary.

3D laser-based photonic full body scans create a detailed surface image of the human body consisting of up to 300 data points per $\mathrm{cm}^{2}$ within 10-12 seconds. The BS technique provides detailed information on whole body or body-part circumferences, surfaces and volumes as well as body height and weight (using an integrated scale) in a way that is not only fast and noninvasive but also comfortable for scanned individuals. Scan data are increasingly used for the acquisition of clinically relevant anthropometric measurements by comparing 3D scan data with data from classical anthropometry (Bretschneider et al. 2009; Koepke et al. 2017b; Lin et al. 2004; Olivares et al. 2007; Wells et al. 2015). Recent studies comparing the body scan technique with classical anthropometry have demonstrated the applicability of the scan technique in an epidemiological context (Jaeschke et al. 2015; Kuehnapfel et al. 2016). In these studies, body scans showed good feasibility, reliability, and validity, and correlations with health parameters 
76 linked to the metabolic syndrome were comparable to studies using manual measurement

77 techniques (Jaeschke et al. 2015).

78

79 One issue with body scan studies are systematic differences between body scan derived 80 measurements and manual measurements, which can be considered as the standard. Height

81 derived from scans is slightly less than manually measured height, and different circumferences

82 are either systematically larger or smaller, when derived from scans, as opposed to manual 83 measurement techniques, in various studies (Domina et al. 2008; Jaeschke et al. 2015; Janssen et 84 al. 2002; Koepke et al. 2017b; Wang et al. 2006). Possible factors influencing such differences 85 are variability of posture, the degree of inspiration, and movement of volunteers while standing 86 in the scanner. Also, there are technical differences between different scanner systems in terms 87 of hardware precision, automatic landmark setting, and measurement output of the scanner 88 89 90 software (Schwarz-Müller et al. 2018). Regarding different body circumferences such as waist or hip circumference, systematic differences between scans and manual measurements can be explained by a slightly tighter fitting or slightly different positioning of the measurement tape, compared to scan derived circumferences (Koepke et al. 2017b; Wang et al. 2006).

92

93 This study aimed to further assess systematic differences between body scans and manual

94 measurements in height, WC, and BMI, with a goal to further validate scan derived 95 measurements in future population based studies. We also aimed to assess the correlation 96 between BMI, derived from different scanner or manual measurements, and total body fat mass, 97 derived from BIA. Similarly, we aim to assess the correlation between WC, derived from 98 different scanner and manual measurements, and visceral fat mass, derived from BIA.

\section{Methods}

As part of a larger research project, a cross-sectional study assessing waist circumference, body

102 height and weight, as well as body composition was conducted on 52 Swiss Armed Forces 103 recruits during their basic training. As this is a purely methodological, and not an epidemiological study, we consider a (homogenous) sample size of 52 to be sufficient in order to show systematic differences between measurement techniques. The study took place in 
107 all male Swiss Armed Forces recruits aged 19-23 (19 years old: 34.6\%, 20 years old: 38.5\%, 21

108 years old: $19.2 \%$, 22 years old: $7.7 \%$, mean age: 20.5 years). Volunteers came from different

109 parts of Switzerland and were not selected for socioeconomic status or other demographic

110 variables. This study was conducted with institutional review board approval (BASEC No. 2016-

111 01625), and participation was voluntary. All volunteers were briefed in written form at the

112 beginning of the study and again orally before the examination. All volunteers signed a detailed

113 informed consent form.

114

115 Body scanner derived measurements were acquired using a semi-mobile Body Scanner

116 (VITUSbodyscan Human Solutions, Kaiserslautern, Germany). This scanner model is equipped

117 with four eye-safe lasers, eight cameras, and acquires up to 300 data points per $\mathrm{cm}^{2}$ as a $3 \mathrm{D}$ point

118 cloud, based on optical triangulation. This type of scanner showed to be reliable and precise in its

119 mesurements (Koepke et al. 2017a). The scanner operating software (Anthroscan, Human

120 Solutions, Kaiserslautern, Germany) calculates more than 150 automatic standard measurements,

121 including height, and a large number of distances and circumferences. The scanner was

122 calibrated according to the manufacturer's instructions at the beginning of the data collection

123 days when body scans and manual measurements were acquired. Volunteers were measured in

124 two positions, once standing straight in an upright position with their feet together (same position

125 as during the manual height measurement with the stadiometer) and another in a standardized

126 position specified by the scanner manufacturer (standing in an upright position, both feet

127 positioned on marks on the scanner platform (spaced approximately $30 \mathrm{~cm}$ apart), arms slightly

128 bent in the elbow and held slightly separated from the body, head in accordance to the Frankfurt

129 Horizontal Plane). All volunteers were briefed before each scan regarding exact positioning on

130 the platform. They were asked to hold their breath after exhalation for the scans, which was

131 about 10 seconds per scan. Volunteers wore only form-fitting underpants and a tight-fitting

132 bathing cap. See Supplemental Figures 1a and $1 \mathrm{~b}$ for the two scan positions.

133

134 For standard WC measurements, a hand-held tape of stretch-resistant quality and automatic 135 retraction was used (seca 201, Seca AG, Reinach, Switzerland). All WC measurements were 136 performed by one of the authors trained in WC measurements (NB) (Koepke et al. 2016; Staub et 137 al. 2018). The tape measurement position was chosen according to WHO guidelines, at midlevel 
138 between the lowest palpable point of the rib cage and the highest palpable point of the iliac crest 139 (WHO 2008). The measurement level was marked with a pen in order to make it visible in 140 subsequently performed body scan acquisitions. Due to a tight schedule given by the Swiss 141 Armed Forces, manual WC measurements were only carried out once for each volunteer. Height 142 measurements were carried out with a standard stadiometer (seca 274, Seca AG, Reinach,

143 Switzerland). Volunteers stood straight in an upright position with their feet together, their back 144 and feet against the stadiometer, and head positioned in accordance with the Frankfurt Horizontal 145 Plane.

146

147 To assess body composition, bioelectric impedance analysis (BIA) was utilized, as time 148 constraints and the need for non-invasive methods did not allow for other assessment methods 149 such as dual-energy X-ray absorptiometry (DXA) or magnetic resonance imaging (MRI). We 150 used a medical 8-point body composition analyzer (Seca mBCA 515, Seca AG, Reinach, 151 Switzerland) measuring weight and calculating the amount of whole-body fat mass and visceral 152 fat mass, muscle mass, and intracellular and extracellular water in the body based on bioelectric 153 impedance measurements across a total of four pairs of electrodes placed on both hands and both 154 feet. Whole body and body part composition was calculated based on mathematical algorithms 155 using the integrated software. This software was validated in different settings and with different 156 ethnic groups (Bosy-Westphal et al. 2017; Bosy-Westphal et al. 2013; Day et al. 2018). For BIA, 157 study volunteers stood barefoot on the two pairs of foot-electrodes and placed each hand on one 158 of the two pairs of hand-electrodes.

159

160

161 162 163 164 165 166 167 168
A dataset using measurements from all described sources was compiled. Height and WC from manual measurements were included. From body scans, height in a straight position and standard position were included. Furthermore, an automatically calculated WC from the scans and a second WC measurement from the scans that was manually adjusted in the software to the WHO measurement point were included. To obtain this second WC scanner measurement, the measurement line of the software was manually adjusted to match the pen mark of the manual WHO measurement level on the scans (see Supplemental Figure 1c). Additionally, weight (from an incorporated electronic scale, Seca AG, Reinach, Switzerland), relative whole-body fat mass $(\%)$, and visceral fat mass (1) from BIA measurements were included. There was one missing 
169 body scan in straight position from one study participant. Therefore, for some statistics, $\mathrm{N}=51$

170 was used instead of $\mathrm{N}=52$.

171

172 Volunteers' BMI was calculated from their weight divided by the square of their height in meters

173 in accordance to WHO guidelines (WHO 2018). Volunteers were classified in different BMI

174 subgroups, volunteers with BMI $<18.5 \mathrm{~kg} / \mathrm{m} 2$ as underweight, volunteers with BMI 18.5 - 24.9

$175 \mathrm{~kg} / \mathrm{m} 2$ as normal weight, volunteers with BMI $\geq 25.0-29.9 \mathrm{~kg} / \mathrm{m} 2$ as overweight, and volunteers

176 with $\mathrm{BMI} \geq 30.0 \mathrm{~kg} / \mathrm{m} 2$ as obese (WHO 2017). In accordance with WHO guidelines, volunteers

177 were categorized for their risk of metabolic complications (RMC) according to their WC. WC <

$17894.0 \mathrm{~cm}$ is associated to a low RMC, WC $94.0-102.0 \mathrm{~cm}$ is associated to an increased RMC,

179 and $\mathrm{WC}>102.0 \mathrm{~cm}$ is associated to a substantially increased RMC (WHO 2008).

180

181 We included the following WC measurements in the study: the manual WC measurement, the

182 automatic scanner measurement in the standard position, and the adjusted scanner measurement

183 at the WHO measurement point. For height, we included the manually measured height, height

184 measured with the scanner in a straight position $(\mathrm{N}=51)$, and height measured with scanner in

185 standard position. We calculated BMI using weight from the internal scale of the BIA and height

186 from the manual measurement as well as using height from the scan in the straight position and

187 the standard position. See Table 1 for an overview of all measurements considered and

188 abbreviations used.

189

190 Statistical analysis

191 Descriptive statistics were calculated for all above-listed measures of WC, height, and BMI. The

192 distributions for WC and BMI were not entirely symmetrical (Supplemental Figure 2), and

193 logarithmic transformation did not considerably change their shape. Therefore, non-parametric

194 and parametric methods were applied to analyse the data. Differences between different WC,

195 height, and BMI measures were tested using Wilcoxon signed-rank tests and paired t-tests. To

196 assess the agreement between the different measurements for WC, height, and BMI, and to

197 compare both measurement methods (scanner vs. manual), Lin's correlations coefficients (CCC)

198 (Lin 1989) were used, Pearson's as well as Spearman rank correlations. Kappa coefficients

199 (Altman 1999) were used to assess the classification agreement according to official WHO BMI 
200 categories for overweight/obesity as well as increased health risks for WC. Agreements,

201 correlations, and intra- and inter-methods comparisons were visualized using scatterplots and

202 Bland Altman Plots (Bland and Altman 1999). For the Bland Altman plots the batplot package in

203 Stata (Version 14.1) was used, which additionally tests the difference between the methods for

204 trend. To compare effect sizes between methods a linear regression was performed to assess the

205 association between BMI and relative whole-body fat mass, divided into BMI subcategories,

206 separately for the different BMI measures. Similarly, a linear regression was performed to assess

207 the association between WC and visceral fat mass, divided into WC subcategories, separately for

208 the different WC measures.

209

\section{2. Results}

211 Visual evaluation of WC measure scatterplots showed an increasing deviation with larger WCs

212 when manually measured WC was compared to automatic scan WC and to manually adjusted

213 scan WC (Figure 1a and 1c). This increasing deviation is also represented as an increasing

214 difference between the measures with an increasing average of the measures (Figure 1b, 1d). The

215 largest difference $(-1.64 \mathrm{~cm}$, paired t-test $\mathrm{p}<0.01)$ between WC measurements was observed

216 between manual WC (mean $81.65 \mathrm{~cm}, \mathrm{SD}=9.04)$ and adjusted scan WC (mean $83.29 \mathrm{~cm}, \mathrm{SD}=$

217 9.95) (Table 2). The smallest and not significant difference $(-0.13 \mathrm{~cm}$, paired t-test $\mathrm{p}=0.376)$ was

218 observed between automatic scan WC (mean $83.15 \mathrm{~cm}$, SD =9.73) and adjusted scan WC (mean

$21983.29 \mathrm{~cm}, \mathrm{SD}=9.95)$. Agreement between automatic scan WC and adjusted scan WC was good

220 (kappa $=0.68$, agreement $=92.88 \%)$ and very good between manual WC and automatic scan WC

$221(\mathrm{k}=0.91$, agreement $=98.08 \%)$. Correlation was very high for all WC measurements $(\mathrm{CCC}>$

222 0.96). For all results see Tables 2-5. The results from the non-parametric tests were very similar

223 (Tables 3 and 5).

224

225 For height, visually there was a constant small difference between manually measured height and

226 standard position scan height as well as between standard position scan height and vertical

227 position scan height. This difference was minimal between manual height and vertical scan

228 height (Figure 2a, 2c, and 2e). The smallest height measurements difference $(+0.20 \mathrm{~cm}$, paired t-

229 test $\mathrm{p}=0.009$ ) was between manual height (mean $178.69 \mathrm{~cm}, \mathrm{SD}=6.82$ ) and straight scan height

230 (mean $178.18 \mathrm{~cm}, \mathrm{SD}=6.55)($ Table 2$)$. The largest difference $(+0.77 \mathrm{~cm}$, paired $\mathrm{t}$-test $\mathrm{p}<0.001)$ 
231 was between manual height (mean $178.69 \mathrm{~cm}, \mathrm{SD}=6.82$ ) and standard scan height (mean

$232177.92 \mathrm{~cm}, \mathrm{SD}=6.78)($ Table 2). There were significant differences between all compared height

233 measurements (Table 2). The correlation was very high for the manually measured height, and

234 both scanned height measurements $(\mathrm{CCC}>0.98)$. The results from the non-parametric tests were

235 again very similar (Tables 2 and 3 ).

236

237 For BMI, the inspection of figure 3 revealed a strong association between BMI calculated with

238 manual height and BMI calculated with scan height in a straight posture. The visual association

239 was slightly deviating for manual BMI versus standard scan BMI, and for standard scan BMI

240 versus straight scan BMI, with increasing BMI (Figure 3a, 3c, 3e). All calculated BMI

241 differences were highly significant (Table 2$)$. The smallest difference $(-0.06$, paired t-test

$242 \mathrm{p}=0.007$ ) was between manual BMI (mean 23.99, SD =4.01) and straight scan BMI (mean 24.09,

$243 \mathrm{SD}=4.06)$. The largest difference $(-0.22$, paired t-test $\mathrm{p}<0.001)$ was between manual BMI (mean

244 23.99, $\mathrm{SD}=4.01)$ and standard scan BMI (mean 24.20, $\mathrm{SD}=4.09)$. The correlation was excellent

245 for all BMI measures (Kappa =1.0, agreement 100\%, CCC >0.99). For all results see Tables 2-5.

246

247 There was a strong correlation between the different BMI measures and relative fat mass

248 (Spearman Rho $>=0.86$, Table 6, Figure 4a). The WHO subcategories of BMI correlated

249 similarly with relative fat mass in all three BMI measures (Figure 5a). Compared to normal

250 weight volunteers, underweight volunteers measured with classic anthropometry showed $-6.6 \%$

251 (95\% CI -13.8 -0.5) less body fat mass. Overweight volunteers showed $11.3 \%$ (CI 95\% 8.2-

252 14.3) more body fat than normal weight volunteers, and obese volunteers showed $20.5 \%$ (CI

$25395 \% 14.5$ - 26.4) more body fat than normal weight volunteers (Figure 5a, upper panel). The

254 results for BMI calculated with scan height in the standard position and in the straight position

255 were very similar. See Figure 5 and supplementary Table 1 for all results.

256

257 There was a strong correlation between the different WC measures and visceral adipose tissue

258 (Spearman Rho $>=0.79$, Table 5), but manual WC deviated increasingly from automatic scan

$259 \mathrm{WC}$ and adjusted scan WC with increasing WC (Figure 4b). For WC subcategories vs. visceral

260 adipose tissue, the correlation was more different for manual WC than for the other WC

261 measures, when WC was more than $102.0 \mathrm{~cm}$ (Figure 5b). Compared to volunteers with WC 
$262<94.0 \mathrm{~cm}$ (low RMC) volunteers with WC 94.0-102.0 cm (increased RMC) measured with 263 classic anthropometry had 1.94 litre (95\% CI 1.53 - 2.34) more visceral fat mass. Volunteers

264 with a WC $>102 \mathrm{~cm}$ (substantially increased RMC) had 4.57 litre (95\% CI 3.7 - 5.44) more

265 visceral fat mass than volunteers with low risk $(\mathrm{WC}<94.0 \mathrm{~cm})$.

266

267 3. Discussion

268 The presented study evaluated whether the positioning of volunteers in the scanner influenced

269 previously observed differences between height measurements derived from body scans and 270 manually measured height. We confirmed that height was less when measured by the scanner, 271 compared to classical anthropometry. When volunteers stood in the scanner in a completely 272 upright position with their feet together, this difference became smaller. Nevertheless, for height 273 measurements, a small difference remained between the scanner and manual classical 274 anthropometry for the same body position.

275

276 Interestingly, other studies found that height was greater in the scanner than measured with 277 classical anthropometry (Jaeschke et al. 2015; Kuehnapfel et al. 2016). Several factors could 278 explain our findings. In classical anthropometry, the stadiometer behind the volunteers may 279 encourage the volunteers to stand straighter than without the stadiometer in the scanner (Koepke 280 et al. 2017b). Additionally, posture variability in the scanner could also have influenced height; 281 in fact, other studies showed relevant differences due to posture variability (Schwarz-Müller et 282 al. 2018; Tomkinson and Shaw 2013; Wells et al. 2015).

283

284 As expected and similarly to other studies, we found an excellent correlation for WC measured 285 by classical manual anthropometry and scanner derived measurements (Koepke et al. 2017b).

286 However, WC was systematically slightly smaller if measured with manual anthropometry than 287 derived from the scanner. A possible explanation for this difference is that volunteers tend to pull 288 their stomach in for manual measurements (Jaeschke et al. 2015). Other studies showed that arm 289 posture had a significant influence on WC measurements (Lennie et al. 2013; Lu et al. 2010). In 290 our study arm posture was different in the scanner (slightly bend elbows and slightly separated 291 from the body, but not too far away in order not to leave the measuring area) as in manual WC, 292 where volunteers had to hold their arms further away from the trunk due to the measurement 
293 procedure. The difference between manual WC and scanner derived measurements was not

294 explainable by a different measuring level, as manual WC deviated more from adjusted scan WC

295 than expected, while there was no significant difference between automatic scan WC and

296 adjusted scan WC. This means that at the population level automatic scan WC is reliable

297 concerning the measuring level and can be equalized to the WHO measuring level.

298

299

Interestingly, manual WC deviated more from scanner measures in obese volunteers compared to

300

301

302

303

304

305

306

307

308

309

310

311

312

313

314

315

316

317

318

319

320

321

322

lean volunteers. Other studies showed low reliability of manual WC measurements in obese subjects (Kuehnapfel et al. 2016; Verweij et al. 2013). It was more challenging to control tape position when WC was larger and more difficult to identify the WHO measurement level in obese volunteers correctly.

The scanner derived measures correlated similarly to total fat and visceral fat as the manually derived measures, probably being even superior when it comes to the correlation between waist circumference and visceral fat in strongly obese persons. As just discussed for waist circumference, this might be due to the difficulty in measuring manually the waist circumference in very obese persons. Overall the scanner offers a reliable tool to assess a number of anthropometric measures in a fast and safe way. Furthermore, as Table 4 showed, the difference in categorizations of BMI and WC between scanner and manual measurements was absent or minimal. The scanner can therefore be regarded as an alternative tool to categorize patients into $\mathrm{BMI}$ and WC categories, if scans are taken for any reason.

Several techniques are used to reconstruct body segment volumes, body segment parameter differences, or segment inertial properties, such as 3D body scanners (Norton et al. 2002), infrared scanners (Smith and Bull 2018), magnetic resonance imaging (Cheng et al. 2000) or dual energy x-ray absorptiometry (DEXA) (Durkin and Dowling 2003). The different techniques have their advantages and disadvantages, depending on the specific research target. For epidemiological research on anthropometrical measures, a 3D full body scanner offers a rapid, safe and reliable alternative.

PeerJ reviewing PDF | (2019:06:38312:1:1:NEW 22 Oct 2019) 
323 This study is the first to compare different postures and different measuring levels to assess

324 height and WC with a 3D body scanner. An advantage for the internal validity of this study was

325 the homogenous study group, namely same age, and sex, which reduced physical inter-individual

326 variability apart from BMI. Limitations for the external validity of this study were the small

327 sample size, and the specificity of the sample, particularly regarding sex and age. Due to time

328 constraints, all measurements were only carried out once per volunteer. One trained researcher

329 did all manual WC measurements. Therefore, we could exclude inter-observer bias, but an intra-

330 observer bias cannot be excluded (Koepke et al. 2017b).

331

\section{4. Conclusion}

333 The body scanner measurement technique seems to be reliable for height and WC and the 334 compared measurement techniques correlated well. However, the small difference in height 335 measurements that also persists after postural correction must be further investigated. Likewise,

336 the observed deviation between manually measured WC and body scan derived values, which 337 systematically increased with increasing BMI of volunteers, must be further investigated.

338 Additionally, extensive studies with volunteers of both sexes, various ages, and different BMIs

339 are required to further evaluate the differences between scanner derived measurements and 340 classic anthropometrics in order to assess the applicability of the scanner technique at a 341 population level. 


\section{Acknowledgements}

346

347 The authors are especially thankful to Andreas Stettbacher (Chief Medical Surgeon), Franz Frey, 348 Alexander Faas, Martino Ghilardi, Marco Müller, and Yvanka Jerkovic from the Swiss Armed

349 Forces for their tremendous (logistic) support. We also thank the IEM collaborators Nikola

350 Koepke, Lena Öhrström, Gulfirde Akgül, Anne Lehner and Nakita Frater for helping to collect

351 the data, and Abagail Breidenstein for English editing.

352

353 
354

355

356

357

358

359

360

361

362

363

364

365

366

367

368

369

370

371

372

373

374

375

376

377

378

379

380

381

382

383

384

385

386

\section{Figure legends}

Figure 1: Associations between different waist circumference measures. A), C) and E) show scatter plots of different WC measures (solid line: $\mathrm{x}=\mathrm{y}$, dotted line: linear regression). B), D) and F) show Bland Altman plots (the solid line including 95\% confidence interval originates from the test for trend using linear regression). There is an increasing deviation between manual measurements and scanner measurements with increasing WC.

Figure 2: Associations between different height measurements. A), C) and E) show scatter plots of different height measures (solid line: $\mathrm{x}=\mathrm{y}$, dotted line: linear regression). B), D) and F) show Bland-Altman Plots (the solid line including 95\% confidence interval originates from the test for trend using linear regression). Panel A) shows the systematic difference between manual height measurement and scanner measurement in the standard scanner position.

Figure 3: Associations between different BMI measurements. A), C) and E) show scatter plots of different BMI measures (solid line: $\mathrm{x}=\mathrm{y}$, dotted line: linear regression). B), D) and F) show Bland Altman plots (the solid line including 95\% confidence interval originates from the test for trend using linear regression).

Figure 4: Associations between BMI and relative fat mass, and between WC and visceral fat mass. A): Associations between BMI and relative fat mass (\%). The different BMI measures are given in different colours and symbols, and linear regression lines. B): Associations between WC and visceral fat mass (1). Different WC measures are given in different colours and symbols, and linear regression lines. The manual measurements are increasingly deviating from the scanner measurements with increasing WC.

Figure 5: coefficients from regression analysis for categories of BMI vs relative fat mass and for categories of WC vs visceral fat mass. A): coefficients and 95\% confidence intervals 
387 from regression analyses of BMI categories and relative fat mass (\%), for the three different 388 measurements. B): coefficients and 95\% confidence intervals from regression analyses of WC 389 categories and visceral fat mass (1), for the three different measurements. The manual WC 390 measurements are increasingly deviating from the scanner measurements with increasing WC.

391

392

393 Supplemental Figure 1: A) standardized position specified by the scanner manufacturer:

394 standing in an upright position, both feet positioned on marks on the scanner platform (spaced 395 approximately $30 \mathrm{~cm}$ apart), arms slightly bent in the elbow and held slightly separated from the 396 body, head in accordance to the Frankfurt Horizontal Plane. B) standing straight in an upright 397 position with feet together (same position as during the manual height measurement with the 398 stadiometer). C) the measurement line of the software was manually moved to match the pen 399 mark of the manual WHO measurement point on the scans.

401

402 Supplemental Figure 2: The distributions for WC, height, and BMI were not entirely 403 symmetrical, neither for manual measurements, nor for the two scanner positions. 
406

407

408

409

410

411

412

413

414

415

416

417

418

419

420

421

422

423

424

425

426

427

428

429

430

431

432

433

434

435

436

437

438

439

440

441

442

443

444

445

446

447

\section{References}

Altman D. 1999. Practical statistics for medical research. Boca Raton: Chapman \& Hall/CRC.

Ashwell M, Gunn P, and Gibson S. 2012. Waist-to-height ratio is a better screening tool than waist circumference and BMI for adult cardiometabolic risk factors: systematic review and meta-analysis. Obes Rev 13(3):275-286.

Bland JM, and Altman DG. 1999. Measuring agreement in method comparison studies. Stat Methods Med Res 8(2):135-160.

Bosy-Westphal A, Jensen B, Braun W, Pourhassan M, Gallagher D, and Muller MJ. 2017. Quantification of whole-body and segmental skeletal muscle mass using phase-sensitive 8-electrode medical bioelectrical impedance devices. European journal of clinical nutrition 71(9):1061-1067.

Bosy-Westphal A, Schautz B, Later W, Kehayias JJ, Gallagher D, and Muller MJ. 2013. What makes a BIA equation unique? Validity of eight-electrode multifrequency BIA to estimate body composition in a healthy adult population. European journal of clinical nutrition 67 Suppl 1:S14-21.

Bretschneider T, Koop U, Schreiner V, Wenck H, and Jaspers S. 2009. Validation of the body scanner as a measuring tool for a rapid quantification of body shape. Skin Res Technol 15(3):364-369.

Cheng CK, Chen HH, Chen CS, Chen CL, and Chen CY. 2000. Segment inertial properties of Chinese adults determined from magnetic resonance imaging. Clin Biomech (Bristol, Avon) 15(8):559-566.

Day K, Kwok A, Evans A, Mata F, Verdejo-Garcia A, Hart K, Ward LC, and Truby H. 2018. Comparison of a Bioelectrical Impedance Device against the Reference Method Dual Energy X-Ray Absorptiometry and Anthropometry for the Evaluation of Body Composition in Adults. Nutrients 10(10).

Domina T, Heuberger R, and MacGillivray M. 2008. Use of 3-dimensional body scans for bodyimage research. Percept Mot Skills 106(2):653-658.

Durkin JL, and Dowling JJ. 2003. Analysis of body segment parameter differences between four human populations and the estimation errors of four popular mathematical models. J Biomech Eng 125(4):515-522.

Jaeschke L, Steinbrecher A, and Pischon T. 2015. Measurement of Waist and Hip Circumference with a Body Surface Scanner: Feasibility, Validity, Reliability, and Correlations with Markers of the Metabolic Syndrome. Plos One 10(3):e0119430.

Janssen I, Heymsfield SB, Allison DB, Kotler DP, and Ross R. 2002. Body mass index and waist circumference independently contribute to the prediction of nonabdominal, abdominal subcutaneous, and visceral fat. American Journal of Clinical Nutrition 75(4):683--688.

Kahn HS, and Bullard KM. 2016. Beyond Body Mass Index: Advantages of Abdominal Measurements for Recognizing Cardiometabolic Disorders. Am J Med 129(1):74-81 e72.

Kjaer IG, Kolle E, Hansen BH, Anderssen SA, and Torstveit MK. 2015. Obesity prevalence in Norwegian adults assessed by body mass index, waist circumference and fat mass percentage. Clin Obes 5(4):211-218. 
448

449

450

451

452

453

454

455

456

457

458

459

460

461

462

463

464

465

466

467

468

469

470

471

472

473

474

475

476

477

478

479

480

481

482

483

484

485

486

487

488

489

Koepke N, Floris J, Bender N, Rühli F, and Staub K. 2016. Waist Circumference und Waist-toHeight-Ratio bei Schweizer Stellungspflichtigen 2016. Bern: Bundesamt für Gesundheit.

Koepke N, Zwahlen M, Wells JC, Bender N, Henneberg M, Ruhli FJ, and Staub K. 2017a. Comparison of 3D laser-based photonic scans and manual anthropometric measurements of body size and shape in a validation study of 123 young Swiss men. PeerJ 5:e2980.

Koepke N, Zwahlen M, Wells JC, Bender N, Henneberg M, Rühli FJ, and Staub K. 2017b. Comparison of 3D laser-based photonic scans and manual anthropometric measurements of body size and shape in a validation study of 123 young Swiss men. PeerJ 5:e2980.

Kuehnapfel A, Ahnert P, Loeffler M, Broda A, and Scholz M. 2016. Reliability of 3D laser-based anthropometry and comparison with classical anthropometry. Scientific Reports 6.

Lam BC, Koh GC, Chen C, Wong MT, and Fallows SJ. 2015. Comparison of Body Mass Index (BMI), Body Adiposity Index (BAI), Waist Circumference (WC), Waist-To-Hip Ratio (WHR) and Waist-To-Height Ratio (WHtR) as predictors of cardiovascular disease risk factors in an adult population in Singapore. PLoS One 10(4):e0122985.

Lennie SC, Amofa-Diatuo T, Nevill A, and Stewart AD. 2013. Protocol variations in arm position influence the magnitude of waist girth. Journal of Sports Sciences 31(12):1353--1358.

Lin JD, Chiou WK, Weng HF, Fang JT, and Liu TH. 2004. Application of three-dimensional body scanner: observation of prevalence of metabolic syndrome. Clinical nutrition (Edinburgh, Scotland) 23(6):1313-1323.

Lin LI. 1989. A concordance correlation coefficient to evaluate reproducibility. Biometrics 45(1):255-268.

Lu J-M, Wang M-JJ, and Mollard R. 2010. The effect of arm posture on the scan-derived measurements. Applied Ergonomics 41(2):236--241.

Murphy J, Bacon SL, Morais JA, Tsoukas MA, and Santosa S. 2019. Intra-Abdominal Adipose Tissue Quantification by Alternative Versus Reference Methods: A Systematic Review and Meta-Analysis. Obesity (Silver Spring, Md) 27(7):1115-1122.

Norton J, Donaldson N, and Dekker L. 2002. 3D whole body scanning to determine mass properties of legs. J Biomech 35(1):81-86.

Olivares J, Wang J, Yu W, Pereg V, Weil R, Kovacs B, Gallagher D, and Pi-Sunyer FX. 2007. Comparisons of body volumes and dimensions using three-dimensional photonic scanning in adult Hispanic-Americans and Caucasian-Americans. J Diabetes Sci Technol 1(6):921-928.

Schwarz-Müller F, Marshall R, and Summerskill S. 2018. Development of a positioning aid to reduce postural variability and errors in 3D whole body scan measurements. Applied Ergonomics 68:90--100.

Smith SHL, and Bull AMJ. 2018. Rapid calculation of bespoke body segment parameters using 3D infra-red scanning. Med Eng Phys 62:36-45.

Staub K, Floris J, Koepke N, Trapp A, Nacht A, Schärli S, Rühli F, and Bender N. 2018. Associations between anthropometric indices, blood pressure, and physical fitness performance in young Swiss men: a cross-sectional study. BMJ open accepted.

Peer) reviewing PDF | (2019:06:38312:1:1:NEW 22 Oct 2019) 
490

491

492

493

494

495

496

497

498

499

500

501

502

503

504

505

506

507
Tomkinson GR, and Shaw LG. 2013. Quantification of the postural and technical errors in asymptomatic adults using direct 3D whole body scan measurements of standing posture. Gait \\& posture 37(2):172--177.

Verweij LM, Terwee CB, Proper KI, Hulshof CTJ, and Mechelen WV. 2013. Measurement error of waist circumference: Gaps in knowledge. Public Health Nutrition 16(2):281--288.

Wang J, Gallagher D, Thornton JC, Yu W, Horlick M, and Pi-Sunyer FX. 2006. Validation of a 3dimensional photonic scanner for the measurement of body volumes, dimensions, and percentage body fat. The American journal of clinical nutrition 83(4):809-816.

Wells JC, Stocks J, Bonner R, Raywood E, Legg S, Lee S, Treleaven P, and Lum S. 2015. Acceptability, Precision and Accuracy of 3D Photonic Scanning for Measurement of Body Shape in a Multi-Ethnic Sample of Children Aged 5-11 Years: The SLIC Study. PLoS One 10(4):e0124193.

WHO. 2008. Waist Circumference and Waist-Hip Ratio. Report of a WHO Expert Consultation. Geneva: WHO.

WHO. 2017. Obesity and overweight. Fact sheet. Geneva: WHO.

WHO. 2018. Body mass index - BMI. http://www.euro.who.int/en/health-topics/diseaseprevention/nutrition/a-healthy-lifestyle/body-mass-index-bmi. 
Table $\mathbf{1}$ (on next page)

Measurements 


\section{Table 1: Measurements}

2

\begin{tabular}{ll}
\hline Used terms & Explanation \\
\hline Manual WC & WC measured with classic manual anthropometry (tape) \\
Automatic scan WC & WC measured with the scanner at automatic scanner position \\
Adjusted scan WC & WC measured with the scanner adjusted at WHO point \\
\hline Manual height & Height measured with classic manual anthropometry (stadiometer) \\
Standard scan height & Height measured with the scanner in standard body posture \\
Straight scan height & Height measured with the scanner in straight body posture \\
\hline Manual BMI & BMI calculated with height from classic manual anthropometry \\
Standard scan BMI & BMI calculated with height from the scanner in standard body posture \\
Straight scan BMI & BMI calculated with height from the scanner in straight body posture \\
\hline
\end{tabular}

3 
Table 2 (on next page)

Descriptive statistics for WC, height, and BMI 
1 Table 2: Descriptive statistics for WC, height, and BMI. A) N, mean, standard deviation

2 (SD), and median. B) Wilcoxon signed rank test, paired t-test.

3

4 A)

\begin{tabular}{lcccc}
\hline & N & Mean & SD & Median \\
\hline WC & & & & \\
Automatic scan WC & 52 & 83.15 & 9.73 & 80.50 \\
Adjusted scan WC & 52 & 83.29 & 9.95 & 81.00 \\
Manual WC & 52 & 81.65 & 9.04 & 80.00 \\
\hline Height & & & & \\
Standard scan height & 52 & 177.92 & 6.78 & 176.10 \\
Straight scan height & 51 & 178.18 & 6.55 & 176.40 \\
Manual height & 52 & 178.69 & 6.82 & 177.25 \\
\hline BMI & & & & \\
Standard scan BMI & 52 & 24.20 & 4.09 & 23.81 \\
Straight scan BMI & 51 & 24.09 & 4.06 & 23.54 \\
Manual BMI & 52 & 23.99 & 4.01 & 23.55 \\
\hline
\end{tabular}

B)

Wilcoxon signed rank

test

Paired t-test

\begin{tabular}{lrrrr}
\hline WC & $\mathbf{Z}$ & $\mathbf{p}$ & Delta (cm) & $\mathbf{p}$ \\
\hline Manual WC vs. scan WC & -4.54 & $<0.001$ & -1.50 & $<0.001$ \\
Manual WC vs. adjusted scan WC & -4.71 & $<0.001$ & -1.64 & $<0.001$ \\
Scan WC vs. adjusted scan WC & -1.06 & 0.295 & -0.13 & 0.376 \\
\hline Height & & & & \\
Manual height vs. standard scan height & 5.56 & $<0.001$ & 0.77 & $<0.001$ \\
Manual height vs. straight scan height & 2.98 & 0.003 & 0.20 & 0.009 \\
Standard scan height vs. straight scan & -5.08 & $<0.001$ & -0.55 & $<0.001$ \\
height & & & & \\
\hline BMI & -5.56 & $<0.001$ & -0.22 & $<0.001$ \\
Manual BMI vs. standard scan BMI & -3.02 & 0.003 & -0.06 & 0.007 \\
Manual BMI vs. straight scan BMI & 5.12 & $<0.001$ & 0.16 & $<0.001$ \\
Standard scan BMI vs. straight scan BMI & & & & \\
\hline
\end{tabular}

5 


\section{Table 3 (on next page)}

Correlations between different measurements of WC, height, and BMI

CCC=Lin's concordance correlation coefficient, $R=$ Pearson's correlation coefficient, $C_{-} b=$ bias correction factor, Rho=Spearman correlational coefficient. 
1 Table 3: Correlations between different measurements of WC, height, and BMI

2 (CCC=Lin's concordance correlation coefficient, $\mathrm{R}=$ Pearson's correlation coefficient, $\mathrm{C}_{-} \mathrm{b}=$ bias

3 correction factor, Rho=Spearman correlation coefficient).

4

\begin{tabular}{|c|c|c|c|c|}
\hline & CCC & $\mathbf{R}$ & C_b & Rho \\
\hline \multicolumn{5}{|l|}{ WC } \\
\hline Manual WC vs. scan WC & 0.964 & 0.979 & 0.985 & 0.964 \\
\hline Manual WC vs. adjusted scan WC & 0.964 & 0.983 & 0.981 & 0.967 \\
\hline Scan WC vs. adjusted scan WC & 0.994 & 0.995 & 1.000 & 0.994 \\
\hline \multicolumn{5}{|l|}{ Height } \\
\hline Manual height vs. standard scan height & 0.988 & 0.995 & 0.993 & 0.989 \\
\hline $\begin{array}{l}\text { Manual height vs. straight scan height } \\
\text { Standard scan height vs. straight scan }\end{array}$ & 0.996 & 0.997 & 0.999 & 0.992 \\
\hline height & 0.992 & 0.996 & 0.996 & 0.992 \\
\hline \multicolumn{5}{|l|}{ BMI } \\
\hline Manual BMI vs. standard scan BMI & 0.997 & 0.999 & 0.998 & 0.998 \\
\hline $\begin{array}{l}\text { Manual BMI vs. straight scan BMI } \\
\text { Standard scan BMI vs. straight scan }\end{array}$ & 0.999 & 0.999 & 1.000 & 0.998 \\
\hline BMI & 0.998 & 0.999 & 0.999 & 0.998 \\
\hline
\end{tabular}

5 


\section{Table 4 (on next page)}

WHO categories of manual BMI, standard scan BMI, and straight scan BMI. WHO categories of manual WC, standard scan WC, and adjusted scan WC 
1 Table 4: WHO categories of manual BMI, standard scan BMI, and straight scan BMI.

2 WHO categories for manual WC, automatic scan WC, and adjusted scan WC

3

\begin{tabular}{lc:lc:lr}
\hline Manual BMI (kg/m2) & $\mathbf{N}$ & $\begin{array}{l}\text { Standard scan BMI } \\
\mathbf{( k g / m 2 )}\end{array}$ & $\mathbf{N}$ & $\begin{array}{l}\text { Straight scan BMI } \\
(\mathbf{k g} / \mathbf{m} 2)\end{array}$ & $\mathbf{N}$ \\
\hline$<18,5$ & 2 & $<18,5$ & 2 & $<18,5$ & 2 \\
$18,5-24,9$ & 32 & $18,5-24,9$ & 32 & $18,5-24,9$ & 32 \\
$25,0-29,9$ & 15 & $25,0-29,9$ & 15 & $25,0-29,9$ & 15 \\
$>=30,0$ & 3 & $>=30,0$ & 3 & $>=30,0$ & 3 \\
\hline Manual WC (cm) & & Automatic scan WC $\mathbf{( c m )}$ & & Adjusted scan WC $\mathbf{( c m )}$ & \\
\hline$<94.0$ & 46 & $<94.0$ & 46 & $<94.0$ & 44 \\
$94.0-101,9$ & 5 & $94.0-101,9$ & 4 & $94.0-101,9$ & 6 \\
$>=102.0$ & 1 & $>=102.0$ & 2 & $>=102.0$ & 2 \\
\hline
\end{tabular}

4 


\section{Table 5 (on next page)}

Kappa coefficients and \% agreement between categories of different measures of WC and $\mathrm{BMI}$ 
1 Table 5: Kappa coefficients and \% agreement between categories of different measures of 2 WC and BMI

3

Kappa \% Agreement

WC

Manual WC vs. automatic scan WC $\quad 0.9081 \quad 98.08 \%$

Manual WC vs. adjusted scan WC

$0.7593 \quad 94.23 \%$

Automatic scan WC vs. adjusted scan WC $\quad 0.681 \quad 92.31 \%$

\section{BMI}

Manual BMI vs. standard scan BMI $\quad 1.000 \quad 100 \%$

Manual BMI vs. straight scan BMI $\quad 1.000 \quad 100 \%$

Standard scan BMI vs. straight scan BMI $\quad 1.000 \quad 100 \%$

4 


\section{Table 6(on next page)}

Pearson's (R) and Spearman (Rho) correlation coefficients between measures of BMI and relative fat mass, and between measures of WC and visceral fat mass 
1 Table 6: Pearson's (R) and Spearman (Rho) correlation coefficients between measures of

2 A) BMI and relative fat mass, and between measures of B) WC and visceral fat mass

3

\begin{tabular}{lll} 
A) Relative Fat Mass & & \\
\hline BMI & R & Rho \\
Manual BMI & 0.884 & 0.863 \\
Straight scan BMI & 0.883 & 0.861 \\
Standard scan BMI & 0.881 & 0.860
\end{tabular}

B) Visceral Adipose Tissue

\begin{tabular}{lll}
\hline WC & R & Rho \\
Manual WC & 0.919 & 0.824 \\
Automatic scan WC & 0.897 & 0.790 \\
Adjusted scan WC & 0.904 & 0.808 \\
\hline
\end{tabular}

4

5 


\section{Figure 1}

Associations between different waist circumference measures
A), C) and
E) show scatter plots of different WC measures. Lines are from linear regressions.
B), D) and F)
F) show Bland Altman plots (
the line including
$95 \%$ confidence interval originates from the test for trend using linear regression). There is an increasing deviation between manual measurements and scanner measurements with increasing WC. 


\section{Waist Circumference}
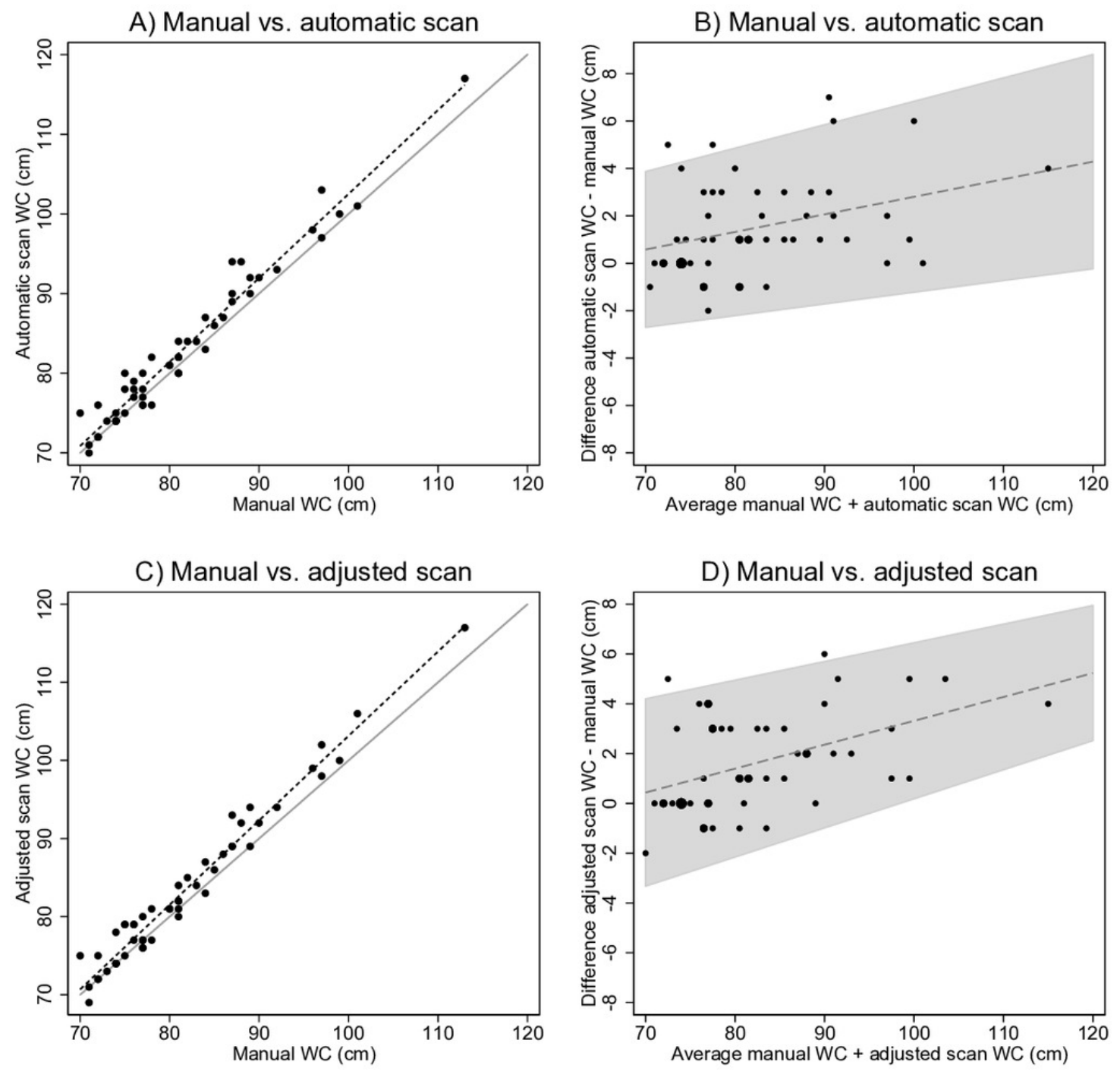

E) Adjusted scan vs. automatic scan
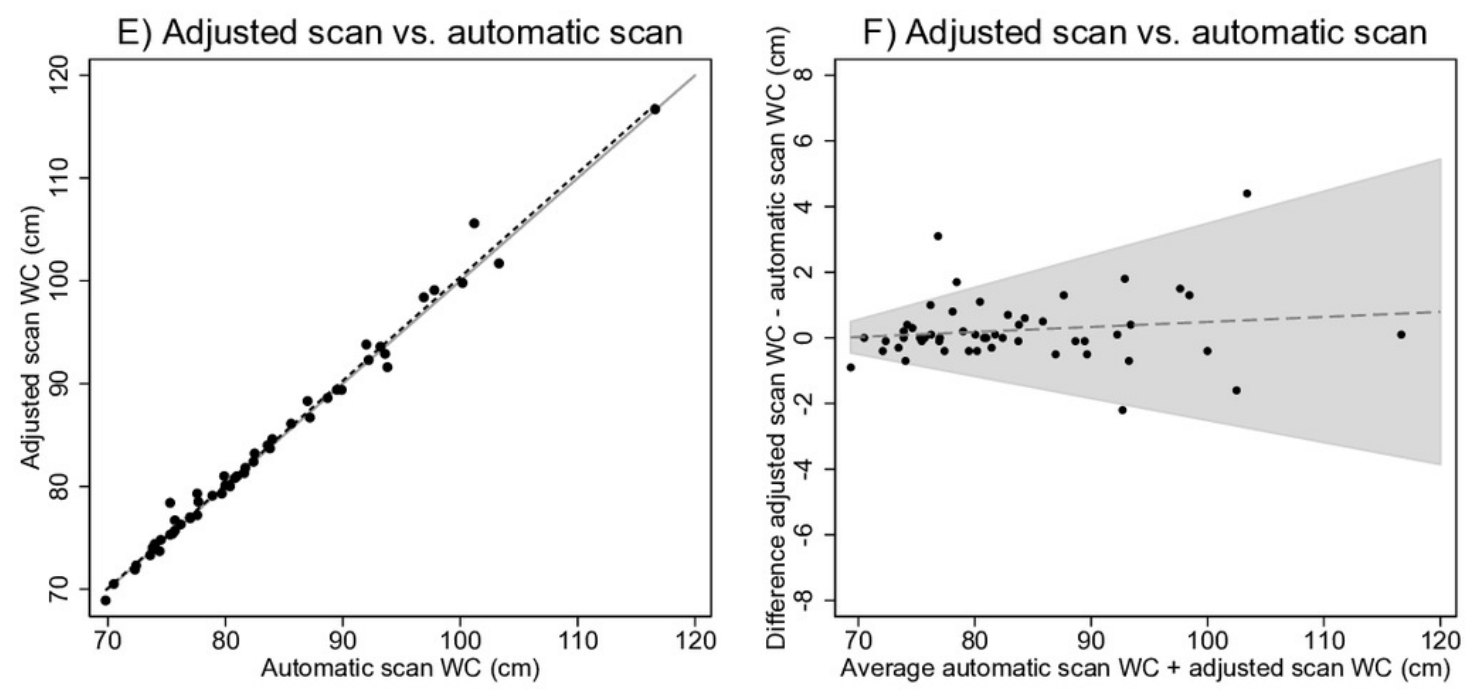


\section{Figure 2}

Associations between different height measurements
A), C) and
E) show scatter plots of different height measures. Lines are from linear

regressions. B), D) and F) show Bland-Altman plots (the line including 95\% confidence interval originates from the test for trend using linear regression). Panel A) shows the systematic difference between manual height measurements and scanner measurements in the standard scanner position. 


\section{Height}

A) Manual vs. standard scan

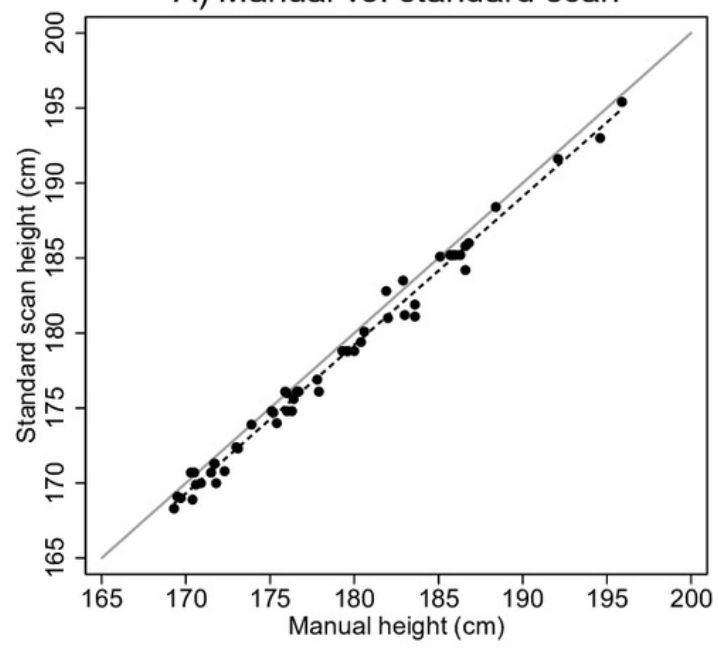

C) Manual vs. straight scan

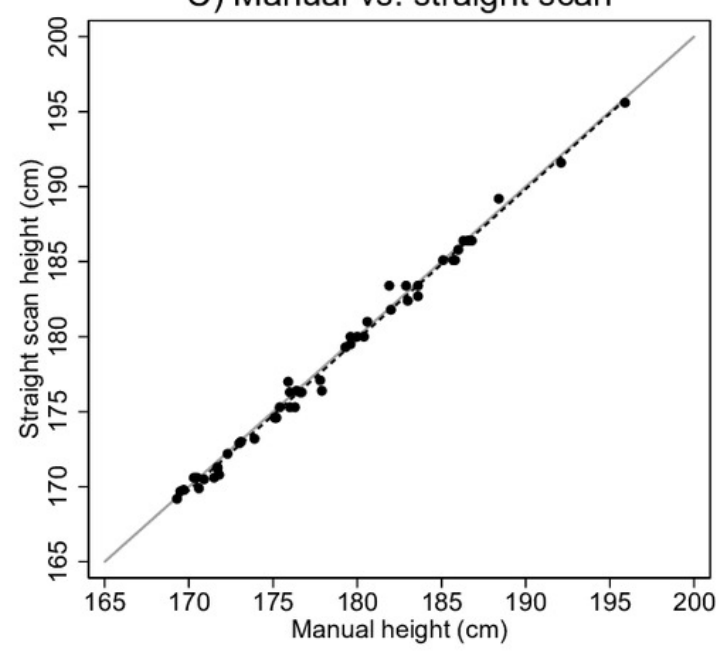

E) Straight scan vs. standard scan

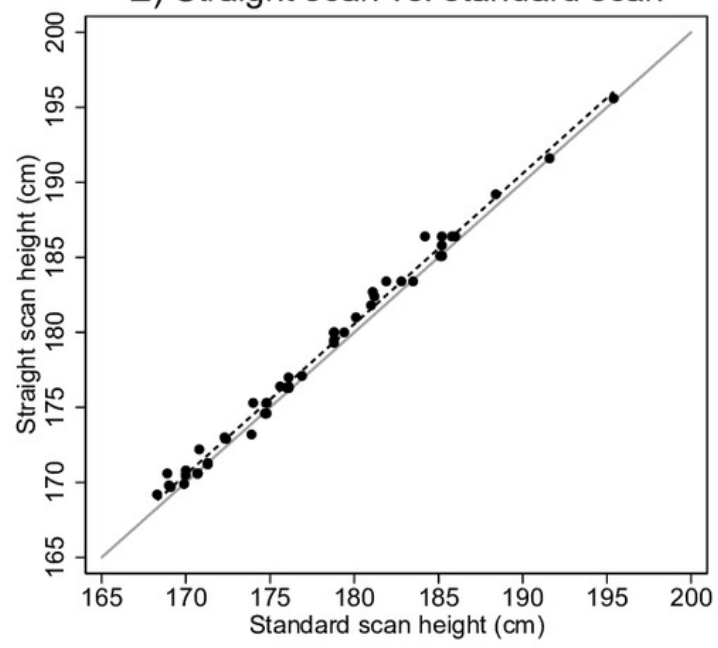

B) Manual vs. standard scan

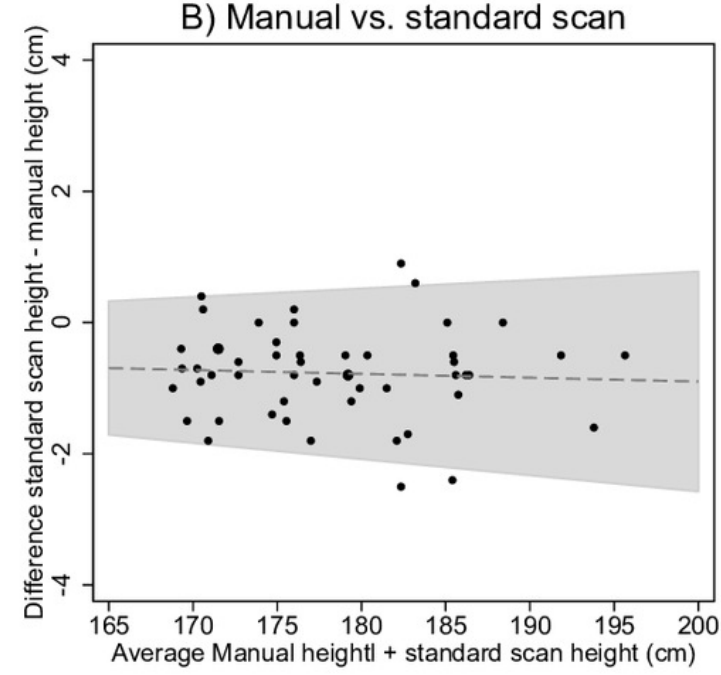

D) Manual vs. straight scan

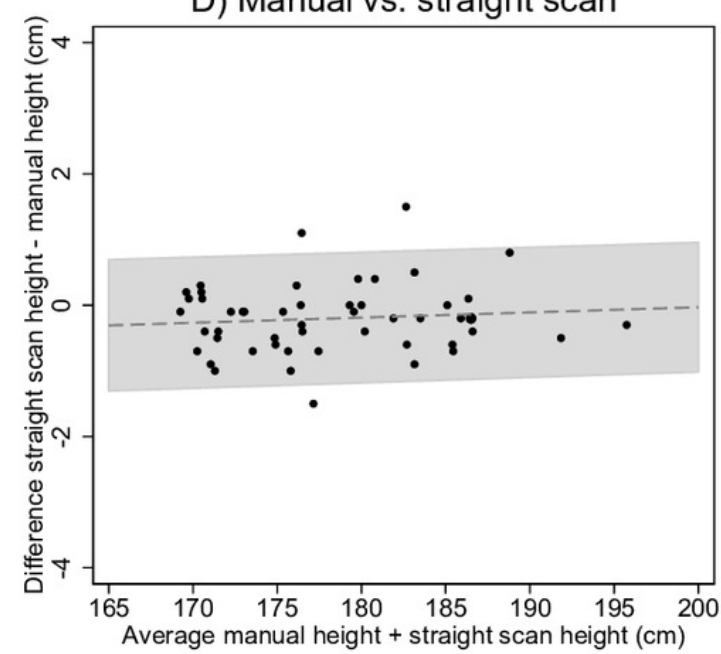

F) Straight scan vs. standard scan

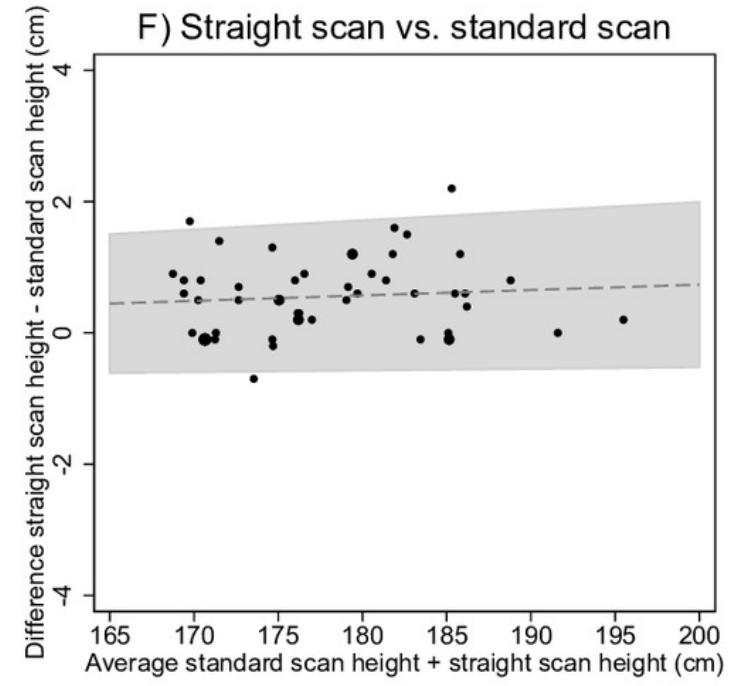


Figure 3

Associations between different BMI measurements

A), C) and E) show scatter plots of different BMI measures. Lines are from linear regressions. B), D) and F) show Bland Altman plots (the line including 95\% confidence interval originates from the test for trend using linear regressions). 


\section{BMI}

A) Manual vs. standard scan

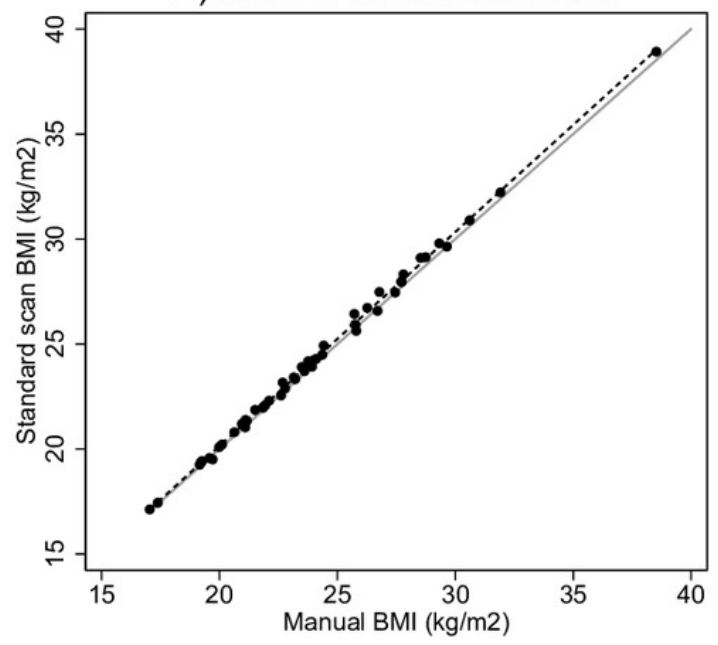

C) Manual vs. straight scant

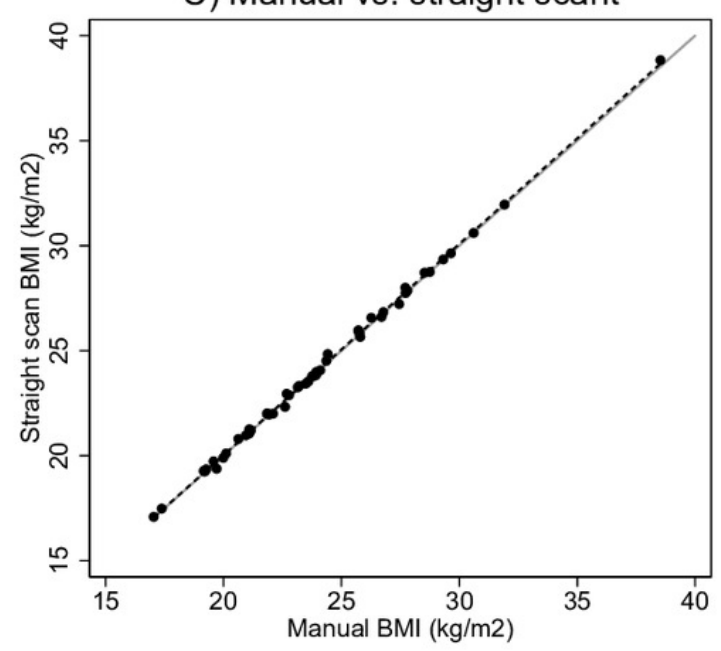

E) straight scant vs. standard scan

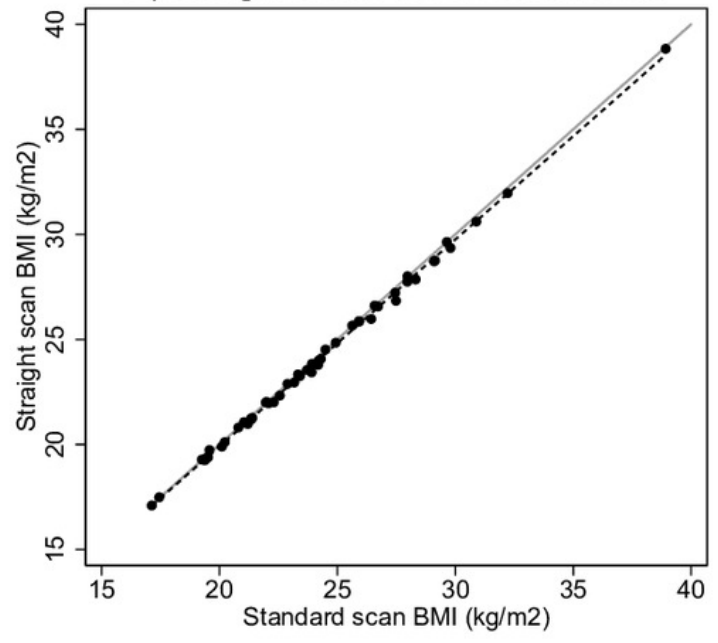

B) Manual vs. standard scan

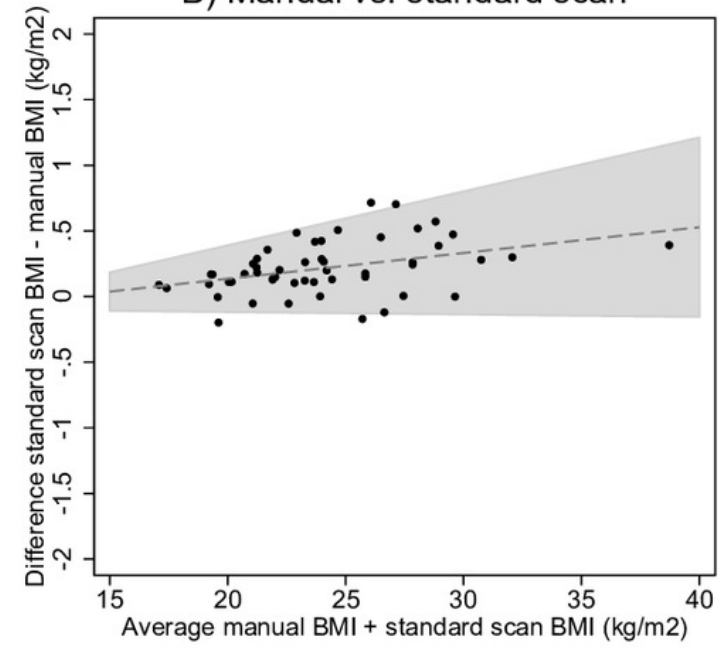

D) Manual vs. straight scan

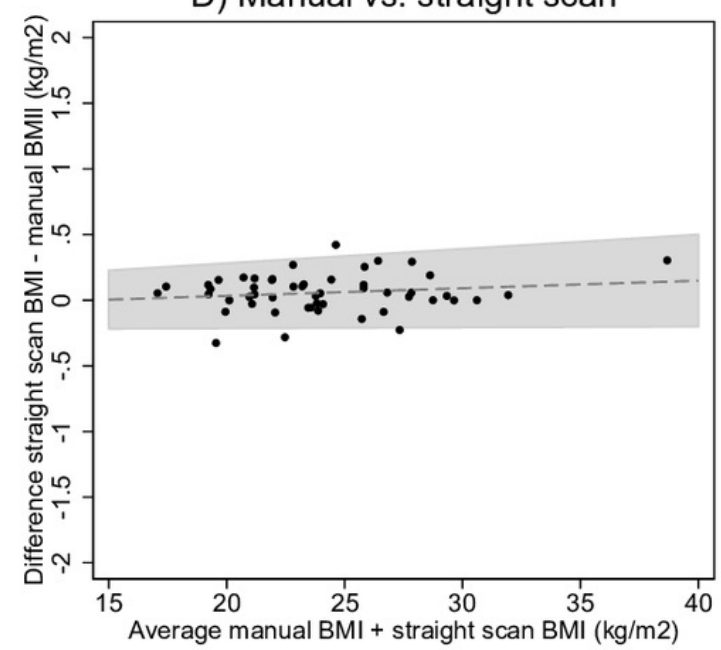

F) straight scan vs. standard scan

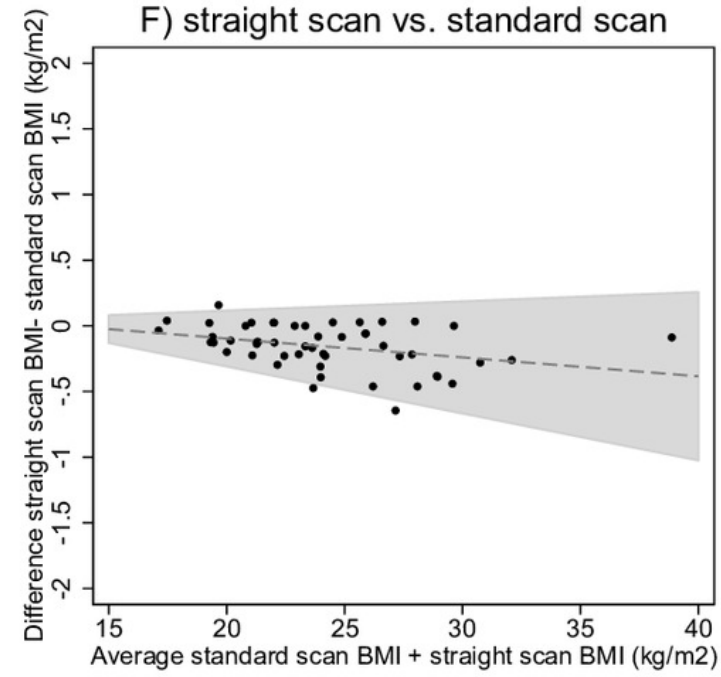


Figure 4

Associations between BMI and relative fat mass, and between WC and visceral fat mass

A: Associations between $\mathrm{BMI}$ and relative fat mass (\%). The different BMI measures are given in different colours nd symbols. B: Associations between WC and visceral fat mass (I). Different WC measures are given in different colours and symbols. The manual measurements are increasingly deviating from the scanner measurements with increasing WC.
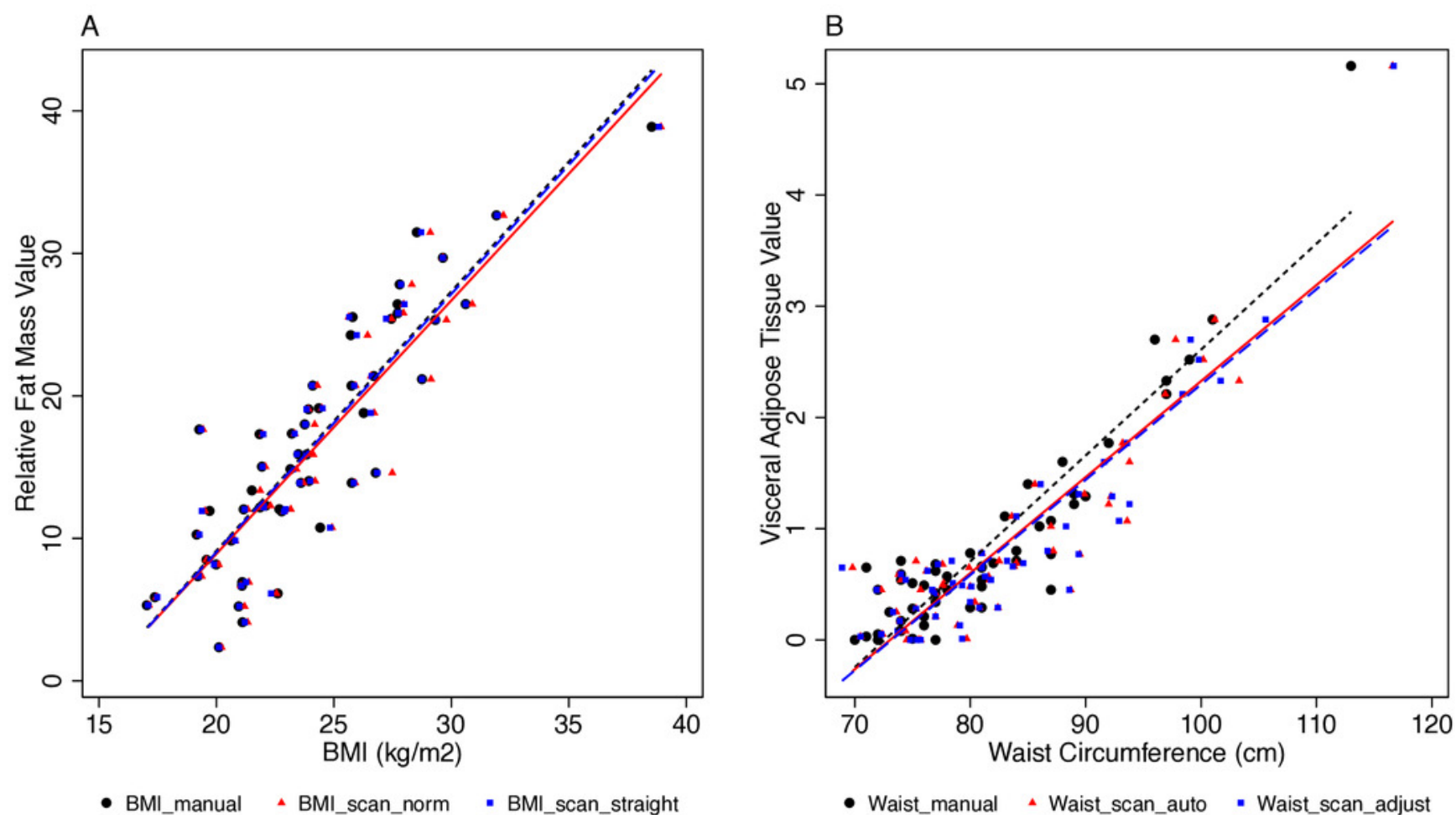

- Waist_manual A Waist_scan_auto - Waist_scan_adjust 


\section{Figure 5}

Coefficients from regression analysis for categories of BMI vs relative fat mass and for categories of WC vs visceral fat mass

A: Coefficients and 95\% confidence intervals from regression analyses of BMI categories and relative fat mass (\%), for the three different measurements. B: Coefficients and $95 \%$ confidence intervals from regression analyses of WC categories and visceral fat mass (I), for the three different measurements. The manual WC measuements are increasingly deviating from the scanner measurements with increasing WC.
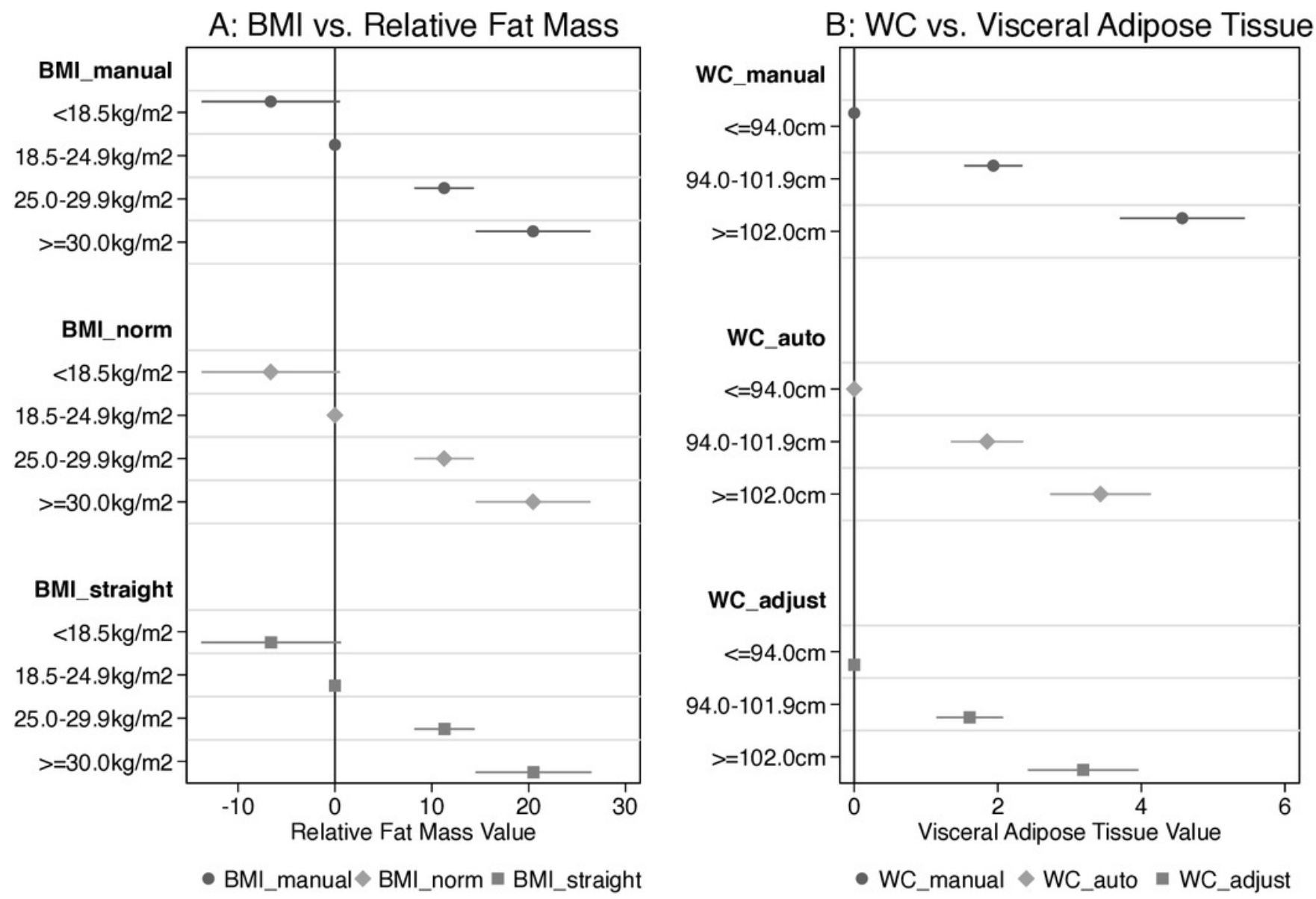